\title{
Intensity-Based Ultrasound Visual Servoing: Modeling and Validation With 2-D and 3-D Probes
}

\author{
Caroline Nadeau and Alexandre Krupa, Member, IEEE
}

\begin{abstract}
In this paper, we present an ultrasound (US) visual servoing to control a robotic system equipped with a US probe. To avoid the difficult and time-consuming image segmentation process, we develop a new approach taking as visual input directly the intensity of the image pixels. The analytic form of the interaction matrix that relates the variation of the intensity features to the motion of the probe is established and used to control the six degrees of freedom (dof) of the robotic system. Our approach is applied with a 2-D and a 3-D US probe, and the results that are obtained with both sensors are compared in simulation. The 2-D probe shows good performances for tracking tasks and the 3-D one, which ensures a larger domain of convergence, is more particularly used for positioning tasks. The intensity-based approach is validated through experimental results performed with a realistic abdominal phantom and with animal soft tissue.
\end{abstract}

Index Terms-Intensity-based control, robotic system, ultrasound (US), visual servoing.

\section{INTRODUCTION}

A LARGE majority of image-guided robotic systems developed for surgical applications are based on a preoperative planning of the gesture and the registration of this planning in the intraoperative environment. Such a strategy concerns for instance orthopedic or neurosurgical robots where a preoperative planning defined by the surgeon is registered using fiducial markers fixed on the rigid surface of the bone or the skull. However with this preoperative control, the gesture is reliable only as long as the patient and the anatomic target remain still. In particular, in operations performed on soft tissues, this strategy is not robust to organs motions and deformations due to physiological motions. An alternative to this strategy is then a visual servoing approach where the robotic system is controlled in real time using intraoperative information provided by a vision sensor. Among the different noninvasive medical imaging modalities,

Manuscript received July 19, 2012; revised December 21, 2012; accepted March 29, 2013. Date of publication April 25, 2013; date of current version August 2, 2013. This paper was recommended for publication by Associate Editor D. Kragic and Editor W. K. Chung upon evaluation of the reviewers' comments. This work was supported by the ANR project US-Comp of the French National Research Agency. This paper was presented in part at the IEEE International Conference on Robotics and Automation, Shanghai, China, May 2011 [1], and in part at the IEEE International Conference on Intelligent Robots and Systems, San Francisco, USA, September 2011 [2].

C. Nadeau is with the CEA List, Saclay 91400, France (e-mail: cnadeau85@ gmail.com).

A. Krupa is with the INRIA Rennes-Bretagne Atlantique, Campus universitaire de Beaulieu, Rennes 35042, France (e-mail: alexandre.krupa@inria.fr).

Color versions of one or more of the figures in this paper are available online at http://ieeexplore.ieee.org.

Digital Object Identifier 10.1109/TRO.2013.2256690 the ultrasound (US) imaging is the only one that provides a visual feedback in real time using a noncumbersome transducer and a nonionizing energy and that can, therefore, be used with no restrictions on the patient, as well as for the surgeon during a medical intervention. Because of such advantages, and despite the low quality of the image it produces, US is a promising imaging modality for image-guided robotic systems.

The previous works dealing with US visual servoing can be classified into two different system configurations. The former, namely eye-to-hand configuration, consists in controlling a surgical instrument using the visual feedback of a fixed US probe. The robotic manipulation offers a better accuracy than the human one and the proposed applications concern needle insertion procedures [3] or cardiac surgery [4]-[6]. The other configuration, namely eye-in-hand configuration, allows the direct control of the US sensor mounted on the robot end-effector for diagnostic purpose [7], [9] or surgical procedure [8].

In order to control 1- to 6-degrees of freedom (dof) of the robotic manipulator, the efficiency of the visual servoing approaches are highly dependent on the choice of appropriate image features. Depending on the configuration, these features can be created by the intersection of the surgical tool with the US beam (eye-to-hand configuration) [3]-[6] or by anatomical landmarks (eye-in-hand configuration) [7]-[9].

In applications where a medical instrument is controlled in order to reach a target, both tool and target are segmented in the US image. In [3], the Hough transform is used to extract the axis of the instrument, rigidly aligned within the US probe plane and an active contour is manually initialized to track a target tumor. Two dof of a needle-insertion robot are then controlled by visual servoing to perform a percutaneous cholecystostomy, while compensating involuntary patient motions. In the same way, in [4], a cross-shaped pattern is used as the target and a passive marker is fixed to the tool. Then, a Radon transform is performed to extract these features in a 3-D US image. However, these transforms are specific for identifying long axes or detecting intersecting lines and can not be extended to detect all kinds of features. In [5], the 4 dof of a surgical forceps inserted in a beating heart through a trocar are controlled by visual servoing, using the two image points that are created by the intersection of the tool with the image plane and segmented using thresholding, morphological filtering and fast labeling process. In relation with this study, in [6], a predictive control scheme was developed to keep the forceps visible in the US image.

In robotic systems where the US probe itself is controlled, which are more particularly within the scope of this study, the image features can only be anatomic ones. In [7], five features 
extraction methods are compared for the tracking of an anatomical point corresponding to the center of an artery in order to servo the in-plane motions of the probe. These methods are based on image similarity measure such as cross correlation and sequential similarity detection or on contour segmentation by a Star [10] or Snake algorithm. For a lithotripsy procedure [8], which consists in the removal of kidney stones using highintensity focused ultrasound (HIFU), two US probes and the HIFU transducer are mounted on the end effector of an $X Y Z$ stage robot to follow a target kidney stone while compensating physiological motions. The translational motions of the robotic effector are controlled with the 3-D position of the kidney stone estimated from its segmentation in two orthogonal US images. Finally, approaches have been proposed to control the 6-dof of the probe with six geometric features built from 2-D moments extracted from a single US image [9] or three orthogonal images [11]. However, the moments computation requires a contour segmentation step whose efficiency depends on the organ shape and is time consuming.

In this paper, we propose a US visual servoing approach based directly on the image intensity for the control of both in-plane and out-of-plane motions of a US probe. The visual features involved in the control law are the intensities of a set of pixels contained in a fixed region of interest of the image. Contrarily to geometric features, the extraction of these intensities does not require any segmentation step, and such a method can, therefore, deal with a large range of anatomic structures with no restriction due to the organ shape or the lack of contours.

Intensity-based features have been recently introduced with success in camera-based visual servoing. In this case, the image formation principle allows the modeling of the interaction between the time variation of these features and the 6 dof of the camera [12]. However, due to the specific geometry of the US sensor, which provides information only along its image plane, the control of the 6 dof of a 2-D US probe requires additional out-of-plane information. In [13], an intensity-based approach to control a 2-D US probe is proposed, using the speckle correlation observed in successive US images to control the out-of-plane motions of the probe. In [14], another intensity-based method is proposed to track the out-of-plane translation of the carotid artery. One dof of the robotic system is then controlled using an interframe block-matching method to identify the artery motion. However, both of these works are position-based and require a reconstruction of the pose of the robot effector from the image measurements.

On the contrary, we present here an image-based visual servoing, where the robot control is directly performed in the image plane, which guarantees a good robustness to calibration errors. In particular, we are able to compute the interaction matrix involved in the control law thanks to the 3-D image gradient. Depending on the geometry of the considered US probe (2-D or $3-\mathrm{D})$, we develop different ways to compute this 3-D gradient, either with derivative filters or with an online estimation algorithm. The 2-D and 3-D approaches are compared and analyzed in simulation environment for positioning and tracking applications, and the first robotic results of this method on animal soft tissue are presented in this paper.
The structure of our paper is as follows. We first introduce the principle of the US visual servoing and insist on the characteristics of the US sensor. We then detail the intensitybased approach with the modeling of the interaction matrix. In Section IV, we focus on the computation of the 3-D image gradient involved in the control law. Solutions to compute this gradient are proposed depending on the geometry of the considered US probe (2-D or 3-D) and an extensive simulation validation is realized to conclude on the advantages of each probe for tracking or positioning tasks. Finally, robotic experiments involving an hybrid force/vision control demonstrate the validity of the approach.

\section{Ultrasound Visual SERVOING}

\section{A. Image-Based Visual Servoing}

The principle of the image-based visual servoing consists in moving a robot so that a set of visual features s extracted from the image provided by a considered vision sensor reaches a set of desired features $\mathbf{s}^{*}$ observed at the desired pose $\mathbf{r}^{*}$ of the robot. The visual servoing control law is designed to minimize the visual error vector defined as $\mathbf{e}(t)=\mathbf{s}(t)-\mathbf{s}^{*}$. In order to try to ensure an exponential decoupled decrease of this error, the classical control law is given by [15]

$$
\mathbf{v}_{p}=-\lambda \widehat{\mathbf{L}}_{\mathbf{s}}^{+}\left(\mathbf{s}(t)-\mathbf{s}^{*}\right)
$$

where $\lambda$ is a positive gain, whose unit is $\mathrm{s}^{-1}$, tuning the decrease of the visual error.

In an eye-in-hand configuration, $\mathbf{v}_{p}$ is the instantaneous velocity that is applied to the visual sensor and $\widehat{\mathbf{L}}_{\mathrm{s}}^{+}$is the pseudoinverse of an estimation of the interaction matrix $\mathbf{L}_{\mathrm{s}}$ that relates the variation of the visual features to the velocity $\mathbf{v}_{p}$ $\left(\dot{\mathbf{s}}=\mathbf{L}_{\mathrm{s}} \mathbf{v}_{p}\right.$ ).

According to [15], the control scheme (1) is known to be locally asymptotically stable when a correct estimation $\widehat{\mathbf{L}_{\mathrm{S}}}$ of $\mathbf{L}_{\mathbf{s}}$ is used (i.e., as soon as $\widehat{\mathbf{L}}_{\mathbf{s}}^{+} \mathbf{L}_{\mathbf{s}}>0$ ).

\section{B. Ultrasound Vision Sensor}

Traditionally, the visual servoing methods refer to vision data that is acquired with a camera mounted on a robotic system. In this case, the vision sensor provides a projection of the 3-D world to a 2-D image and a set of 2-D image features can be used to control the 6 dof of the system. In the particular case of 2-D US visual servoing, the image formation principle and the geometry of the vision sensor are far different from the ones of a camera.

1) US Sensor Model: With a 2-D US probe, the created image corresponds to a cross section of the visual target (see Fig. 1). Therefore, only the physical points lying in the US beam are represented in the US image.

Let the image plane, or probe plane, be defined by the beam of US waves emitted by the US sensor. The probe frame $\mathscr{F}_{p}$ is represented in Fig. 1, its origin is attached to the image center $\left(u_{0}, v_{0}\right), \mathbf{y}_{\mathbf{p}}$ is aligned with the propagation direction of the US, and $\mathbf{z}_{\mathbf{p}}$ is orthogonal to the image plane. With this convention, 

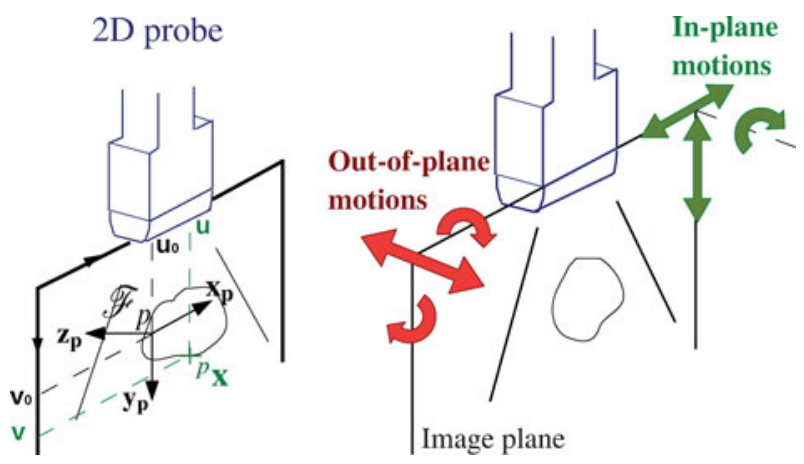

Fig. 1. Contrarily to a projective camera that gives a 2-D projection of the 3-D world, a 2-D US probe provides visual information only within its image plane, making controlling its out-of-plane motions difficult.

the geometric model that relates the 3-D coordinates of a physical point ${ }^{p} \mathbf{X}=\left({ }^{p} X,{ }^{p} Y,{ }^{p} Z\right)$ belonging to the probe plane $\left({ }^{p} Z=0\right)$ with the pixel coordinates $(u, v)$ of the corresponding image point is such as

$$
\begin{cases}u=k_{u} & { }^{p} X+u_{0} \\ v=k_{v} & { }^{p} Y+v_{0}\end{cases}
$$

where the scale factors $\left(k_{u}, k_{v}\right)$ allow the conversion from metric coordinates to pixel coordinates. We can, therefore, define the respective scale factors $\left(s_{x}=1 / k_{u}, s_{y}=1 / k_{v}\right)$ that convert the pixel coordinates to metric coordinates and correspond to the intrinsic parameters of the probe. With the extrinsic parameters of the probe that characterize its pose in a given reference frame, these intrinsic parameters are used to determine, for each image point, the 3-D coordinates of the corresponding physical point. Both intrinsic and extrinsic parameters are estimated by a calibration procedure [16].

2) Ultrasound Wave Physics: The US wave is a mechanical wave that generates pressure variations in the medium in which it travels. At the interface between two mediums of different impedances, the wave is partly transmitted and partly reflected toward the probe. The value of the reflected echo depends on the relative values of the acoustic impedances of both mediums. Moreover, when the incident ultrasonic wave is not orthogonal to the interface, both reflected and refracted waves are produced. The angle of the transmitted US beam depends on the angle of the incident beam and on the propagation velocity of the US in both mediums.

During their propagation in homogeneous materials, the US waves are also subject to two additional physics phenomena, the attenuation and the diffraction. The former depends on the distance to the US source and on an absorption linear coefficient of the medium. The latter occurs when the interface hit by the US beam is small with respect to the US wavelength. The wave energy is then reflected in every direction, which creates a granular pattern in the US image called speckle.

3) B-Mode Image: The US signal can be easily represented by the amplitude of the echo that is reflected by interfaces it goes through. This representation mode, namely A-mode, allows the visualization of a single US line since the echo amplitude is given as a function of the distance to the source.
Currently, the more utilized representation of the US signal is the B-mode representation that associates a gray level value (between 0 and 255) with the US echo amplitude. This representation allows the conservation of the spatial disposition of the US beams and the reconstructed B-mode image shows all US lines emitted by the probe. Compared with A-mode images, several processing are applied to the US signal, namely, the attenuation compensation with an adaptive gain, the data interpolation to fill the full 2-D image and a logarithmic compression of the signal.

Despite the physical characteristics of the US sensor, which is based on the propagation and reflection of acoustic waves, we treat it as a vision sensor since it provides gray-scale images in its common B-mode representation.

\section{INTENSITY-BASED APPROACH}

The specificity of the US sensor in terms of geometry and image formation implies new challenges for the visual control compared with the camera-based approach. While light changes and depth estimation are no more an issue with the US modality, the major difficulties consist in the processing of the images and in the control of the out-of-plane motions of the sensor.

\section{A. Intensity Features}

In this study, we propose to address the first difficulty linked to the US image low quality and to the real-time constraint by avoiding any segmentation step and considering as visual features $s$ the intensity values of the pixels of a region of interest (ROI) of the US image:

$$
\mathbf{s}=\left(I_{1,1}, \ldots, I_{u, v}, \ldots, I_{M, N}\right)
$$

where $M$ and $N$ are, respectively, the width and the height of the ROI, and $I_{u, v}$ represents the intensity of the pixel of 2-D coordinates $(u, v)$ in the US image.

\section{B. Modeling of the Interaction Matrix}

The second challenge is addressed by the computation of the interaction matrix $\mathbf{L}_{\mathrm{s}}$ that links the variation of these intensity features to both in-plane and out-of-plane motion of the US probe in order to control the 6 dof of the probe. The objective is, therefore, to determine the analytic form of the time variation of one pixel intensity in the US image as a function of the probe velocity $\mathbf{v}_{\mathbf{c}}$, that is $\mathbf{L}_{I_{u, v}}$ such as $\dot{I}_{u, v}=\mathbf{L}_{I_{u, v}} \mathbf{v}_{p}$.

Letting the probe control frame $\mathscr{F}_{p}\left(\mathbf{x}_{\mathbf{p}}, \mathbf{y}_{\mathbf{p}}, \mathbf{z}_{\mathbf{p}}\right)$ be the frame attached to the center of the US image where $\left(\mathbf{x}_{\mathrm{p}}, \mathbf{y}_{\mathrm{p}}\right)$ defines the image plane and where $z_{p}$ corresponds to the elevation axis, the 3 -D coordinates ${ }^{p} \mathbf{x}_{P}=\left({ }^{p} x_{P},{ }^{p} y_{P},{ }^{p} z_{P}\right)$ of the point $P$ attached to the probe that corresponds to the pixel $p_{x}$ of coordinates $(u, v)$ in the image are such that

$$
\left(\begin{array}{c}
{ }^{p} x \\
{ }^{p} y \\
{ }^{p} z
\end{array}\right)=\left(\begin{array}{c}
s_{x}\left(u-u_{0}\right) \\
s_{y}\left(v-v_{0}\right) \\
0
\end{array}\right)
$$

where ${ }^{p} z_{P}=0$ since the point $P$ belongs to the image plane.

The intensity $I_{u, v}(t)$ of the pixel $p_{x}$ in the B-mode image acquired by the US probe at the time $t$ corresponds to the 


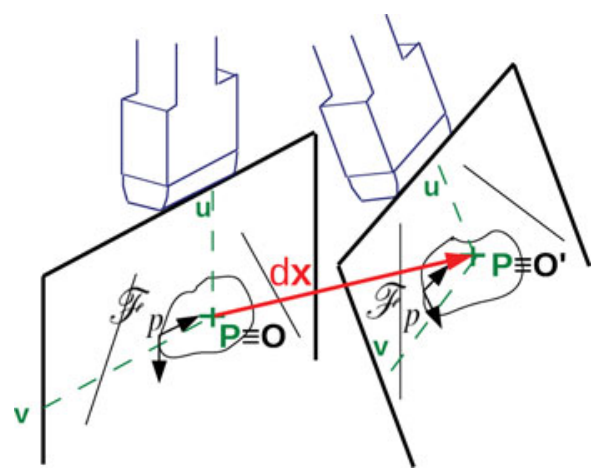

Fig. 2. Under the hypothesis of a moving probe in a motionless environment, the image point $P$ of constant coordinates $(u, v)$ in the US image coincides with a 3-D point $O$ at time $t$ and with a different 3-D point $O^{\prime}$ at time $t+d t$.

amplitude $I_{\mathrm{US}}\left(\mathbf{x}_{O}, t\right)$ of the US echo reflected by a physical point $O$ of coordinates $\mathbf{x}_{O}$ belonging to the observed object

$$
I_{u, v}(t)=I_{\mathrm{US}}\left(\mathbf{x}_{O}, t\right) .
$$

Under the hypothesis (HI) that the probe is moving in a motionless environment, at time $t+d t$ the point $P$, rigidly attached to the probe frame, coincides with a different physical point $O^{\prime}$ (see Fig. 2). The 3-D coordinates of $O^{\prime}$ are $\mathbf{x}_{O}+\mathbf{d x}$, where $\mathrm{dx}$ is the displacement of $P$ due to the probe motion during the time $d t$. The intensity of the pixel $p_{x}$ at time $t+d t$ is then equal to the US echo reflected by $O^{\prime}$ :

$$
I_{u, v}(t+d t)=I_{\mathrm{US}}\left(\mathbf{x}_{O}+\mathbf{d x}, t+d t\right) .
$$

The computation of the interaction matrix is based on the hypothesis (H2) of the constancy of the US wave reflection by a given physical structure. Under this assumption, the US echo reflected by a physical point is independent of the time

$$
I_{\mathrm{US}}\left(\mathbf{x}_{O}, t\right)=I_{\mathrm{US}}\left(\mathbf{x}_{O}, t+d t\right)=I_{\mathrm{US}}\left(\mathbf{x}_{O}\right) .
$$

This is a strong hypothesis since the intensity of the US echo reflected by an organ interface is dependent on the relative orientation of the US probe to this interface, but this conservation equation is a posteriori validated by the results that are obtained with an abdominal phantom and animal soft tissue (see Section VI). With (H2), we can combine (5) and (6) as follows:

$$
I_{u, v}(t+d t)-I_{u, v}(t)=I_{\mathrm{US}}\left(\mathbf{x}_{O}+\mathbf{d x}\right)-I_{\mathrm{US}}\left(\mathbf{x}_{O}\right) .
$$

By approximating $I_{\mathrm{US}}$ by its first-order Taylor polynomial around $\mathrm{x}_{O}$, it becomes

$$
\begin{aligned}
I_{\mathrm{US}}(\mathbf{x}) \approx & I_{\mathrm{US}}\left(\mathbf{x}_{O}\right)+\frac{\partial I_{\mathrm{US}}\left(\mathbf{x}_{O}\right)}{\partial x}\left(x-x_{O}\right) \\
& +\frac{\partial I_{\mathrm{US}}\left(\mathbf{x}_{O}\right)}{\partial y}\left(y-y_{O}\right)+\frac{\partial I_{\mathrm{US}}\left(\mathbf{x}_{O}\right)}{\partial z}\left(z-z_{O}\right) .
\end{aligned}
$$

We set $\mathbf{x}=\mathbf{x}_{O}+\mathbf{d} \mathbf{x}$ with $\mathbf{d} \mathbf{x}=(d x, d y, d z)$ to obtain

$$
I_{u, v}(t+d t)-I_{u, v}(t) \approx \frac{\partial I_{\mathrm{US}}}{\partial x} d x+\frac{\partial I_{\mathrm{US}}}{\partial y} d y+\frac{\partial I_{\mathrm{US}}}{\partial z} d z .
$$

We divide (9) by $d t$ to express the time variation of $I_{u, v}$ as a function of the motion ${ }^{p} \dot{\mathbf{x}}_{P}$ of the image point $P$ with respect to the environment and expressed in $\mathscr{F}_{p}$ :

$$
\dot{I}_{u, v} \approx \nabla I_{(u, v)}{ }^{p} \dot{\mathbf{x}}_{P}
$$

with $\nabla I_{u, v}=\left[\begin{array}{lll}\nabla I_{x} & \nabla I_{y} & \nabla I_{z}\end{array}\right]$ the 3-D image gradient associated to the pixel $(u, v)$. It is constituted of three components $\nabla I_{x}=\frac{\partial I}{\partial x}, \nabla I_{y}=\frac{\partial I}{\partial y}$, and $\nabla I_{z}=\frac{\partial I}{\partial z}$ that describe the intensity variation of the pixel $(u, v)$ along the three axes of the image frame.

In the frame of the US probe $\mathscr{F} p$, the velocity of the point $P$ attached to the probe, with respect to the environment, is linked to the instantaneous velocity of the US probe $\mathbf{v}_{\mathbf{c}}=\left({ }^{p} \nu,{ }^{p} \omega\right)$, according to Varignon's formula of velocity composition in a solid [18]

$$
{ }^{p} \dot{\mathbf{x}}_{P}=\left({ }^{p} \nu-\left[{ }^{p} \mathbf{x}_{P}\right]_{\times}{ }^{p} \omega\right)
$$

that can be rewritten as

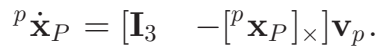

From (10), (12), and ${ }^{p} z=0$, the interaction matrix $L_{I_{u, v}}$ of size $1 \times 6$ associated with the visual feature $I_{u, v}$ is written as

$$
L_{I_{u, v}}=\left[\begin{array}{llllll}
\nabla I_{x} & \nabla I_{y} & \nabla I_{z} & y \nabla I_{z} & -x \nabla I_{z} & x \nabla I_{y}-y \nabla I_{x}
\end{array}\right]
$$

and the complete interaction matrix $\mathbf{L}_{\mathrm{s}}$ is built by stacking the $M \times N$ matrices $L_{I_{u, v}}$

$$
\mathbf{L}_{\mathbf{s}}=\left(\begin{array}{c}
L_{I_{1,1}} \\
\vdots \\
L_{I_{M, N}}
\end{array}\right) .
$$

\section{COMPUTATION OF THE IMAGE GRADIENT}

To control the 6 dof of the US probe, the variation of the visual features is related to both in-plane and out-of-plane motions of the probe. In the interaction matrix, this variation is dependent on the 3-D image gradient that has to be known. Solutions to compute this information are proposed here, depending on the geometry of the considered US probe.

\section{A. With a Classical 2-D Probe}

1) 3-D Filter: In image processing, the image gradient is commonly estimated using directional image filters like the Sobel ones in the case of 2-D gradient that separately compute the horizontal and vertical derivatives of the image. These filters are based on two separable operators: a smoothing operator perpendicular to the derivative direction and a central difference in the derivative direction [18]. In the extended case of 3-D image gradient, three filters can be designed on the same model to estimate the image gradient components $\left(\nabla I_{x}, \nabla I_{y}, \nabla I_{z}\right)$. Using two additional parallel images around the current one, a $3 \times 3 \times 3$ filter can be applied along each direction to compute these three components (see Fig. 3).

With a conventional 2-D US probe, a small back and forth translational motion along the elevation direction is required to capture the additional images needed to compute the gradient components with the 3-D filters. Because of this limitation, the gradient computation described previously is dedicated more 


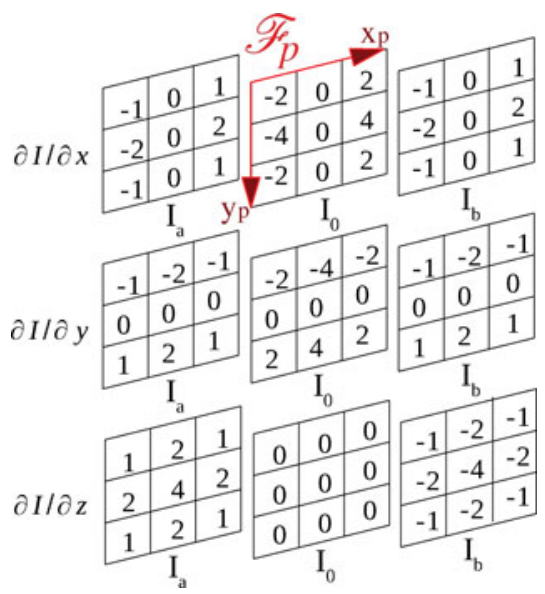

Fig. 3. Three filters are applied on each pixel of the current image $I_{0}$ to compute the gradient components $\left(\nabla I_{x}, \nabla I_{y}, \nabla I_{z}\right)$ using the additional parallel images $I_{a}$ and $I_{b}$ acquired on both sides of $I_{0}$.

specifically to tracking tasks where the initial image is considered as the desired one, and the visual task consists in the automatic stabilization of this image by compensating rigid motions of the target. During such a tracking task, the US probe remains close to its desired pose and the interaction matrix can be estimated at the desired pose of the probe, with the back and forth motion, without being updated during the servoing.

2) Online Gradient Estimation: We also propose an approach to estimate online the image gradient without additional motions of the probe to acquire parallel images. For this purpose, we distinguish the in-plane components of the gradient $\nabla I_{x}, \nabla I_{y}$ from the out-of-plane component $\nabla I_{z}$. The former are directly computed in the current US image with a 2-D Sobel derivative filter, while the latter is estimated from the current image and the previous ones acquired during the motion of the probe. This estimation is based on the approximation given by the Taylor expansion of the intensity of a 3-D point of coordinates $\mathbf{x}=(x, y, z)$ :

$$
I_{U S}(\mathbf{x}+\mathbf{d x}) \approx I_{U S}(\mathbf{x})+\nabla I_{x} d x+\nabla I_{y} d y+\nabla I_{z} d z .
$$

Let $O(x, y, z)$ be the physical point coinciding with the image point $P$ of pixel coordinates $(u, v)$ at time $t$. If the probe moves, at time $t+d t$ the image point $P(u, v)$ coincides with a different physical point $O^{\prime}\left(x^{\prime}, y^{\prime}, z^{\prime}\right)$. We have then the equality between the intensity of the US echo $I_{\mathrm{US}}$ reflected by $O$ (respectively, $\left.O^{\prime}\right)$ and the intensity of the pixel $P$ of the US image acquired at time $t$ (respectively, $t+d t$ ):

$$
\left\{\begin{array}{l}
I_{\mathrm{US}}(x, y, z)=I_{u, v}(t) \\
I_{\mathrm{US}}\left(x^{\prime}, y^{\prime}, z^{\prime}\right)=I_{u, v}(t+d t)
\end{array}\right.
$$

We can express the coordinates of the physical points in the frame of the probe at time $t$ referred to as $\mathscr{F}_{p}$ :

$$
\left(\begin{array}{c}
{ }^{p} x \\
{ }^{p} y \\
{ }^{p} z
\end{array}\right)=\left(\begin{array}{c}
s_{x}\left(u-u_{0}\right) \\
s_{y}\left(v-v_{0}\right) \\
0
\end{array}\right)
$$

and

$$
\left(\begin{array}{c}
{ }^{p} x^{\prime} \\
{ }^{p} y^{\prime} \\
{ }^{p} z^{\prime}
\end{array}\right)={ }^{p} \mathbf{R}_{p^{\prime}}\left(\begin{array}{c}
s_{x}\left(u-u_{0}\right) \\
s_{y}\left(v-v_{0}\right) \\
0
\end{array}\right)+{ }^{p} \mathbf{t}_{p^{\prime}} .
$$

The rotation matrix ${ }^{p} \mathbf{R}_{p^{\prime}}$ and the translation vector ${ }^{p} \mathbf{t}_{p^{\prime}}$ describing the pose of the probe at time $t+d t$ expressed in the frame of the probe at time $t$ are given by the robot odometry. Considering a small motion of the probe, $O^{\prime}$ is close to $O$, and we can apply (15)

$$
\begin{aligned}
I_{\mathrm{US}}\left({ }^{p} x^{\prime},{ }^{p} y^{\prime},{ }^{p} z^{\prime}\right)= & I_{\mathrm{US}}\left({ }^{p} x,{ }^{p} y, 0\right)+\nabla I_{x}\left({ }^{p} x^{\prime}-{ }^{p} x\right) \\
& +\nabla I_{y}\left({ }^{p} y^{\prime}-{ }^{p} y\right)+\nabla I_{z}{ }^{p} z^{\prime} .
\end{aligned}
$$

With the relationship (16), this equation can be rewritten as follows:

$$
\begin{aligned}
I_{u, v}(t+d t)= & I_{u, v}(t)+\nabla I_{x}\left({ }^{p} x^{\prime}-{ }^{p} x\right)+\nabla I_{y}\left({ }^{p} y^{\prime}-{ }^{p} y\right) \\
& +\nabla I_{z}{ }^{p} z^{\prime} .
\end{aligned}
$$

In this equation, the out-of-plane component of the gradient $\nabla I_{z}$ is related to a set of known values. The pixel intensities at time $t$ and $t+d t$ and the in-plane gradient components, computed at time $t+d t$, are measured in the US image.

Equation (20) involves image measurements and is therefore sensitive to image noise. To ensure a better robustness of the gradient estimation, we implement a least squares method to compute $\nabla I_{z}$ from a set of several data.

The parametric model of the considered system (20) is rewritten in discrete time as

$$
Y_{[k+1]}=\Phi_{[k]} \theta_{[k]}+b_{[k+1]}
$$

with

$$
\left\{\begin{array}{l}
Y_{[k+1]}=\left(I_{u, v[k+1]}-I_{u, v[k]}-\nabla I_{x[k]} d x_{[k]}-\nabla I_{y[k]} d y_{[k]}\right) \\
\Phi_{[k]}=d z_{[k]} \\
\theta_{[k]}=\nabla I_{z_{[k]}}
\end{array}\right.
$$

where $\theta$ is the parameter of the system to estimate, $Y$ and $\Phi$ are measured at each iteration, and since we have no a priori on the model noise, we choose $b$ as a white noise term to represent the image noises and robot measure errors.

With the parametric representation, the output of the system can be a priori predicted $\widehat{Y}_{[k+1]}$ from the parameter estimate $\widehat{\theta}_{[k]}$ :

$$
\widehat{Y}_{[k+1]}=\Phi_{[k]} \widehat{\theta}_{[k]} .
$$

The a priori error between this prediction and the real value of the system output $E_{[k+1]}$ is then defined as

$$
\begin{aligned}
E_{[k+1]} & =Y_{[k+1]}-\widehat{Y}_{[k+1]} \\
& =Y_{[k+1]}-\Phi_{[k]} \widehat{\theta}_{[k]} .
\end{aligned}
$$

The aim of the least-squares approach is to estimate the parameter $\theta$ that minimizes a criterion defined as the quadratic sum of 
the prediction errors $E_{[k+1]}$ obtained at each iteration

$$
J\left(\hat{\theta}_{[k]}\right)=\sum_{j=0}^{k} \beta^{k-j}\left(Y_{[j]}-\Phi_{[j]} \widehat{\theta}_{[k]}\right)^{2} .
$$

The scalar $\beta(0<\beta<1)$ is a weighting factor, also called the forgetting factor, used to lower the importance of past data.

Several approaches have been proposed to solve this minimization, and among these, we can distinguish nonrecursive strategies from recursive ones.

Indeed, the limitations of the recursive algorithms are underlined in [19]. The stability of these algorithms is not guaranteed without the introduction of a dead zone or a stabilization term when considering noises and disturbances. At the same time, the improvement brought by these modifications in terms of robustness is done at the expense of the algorithm efficiency. Finally, when dealing with noised signals, direct identification methods are shown to offer better robustness and results [19].

We propose, therefore, to consider a sliding least-squares algorithm, where the estimate of the parameter $\hat{\theta}_{[k]}$ is computed using a set of measures $Y$ and $\Phi$ acquired on a window of size $N_{\mathrm{LS}}$. This estimate is defined in [19] for the multidimensional case and can be written as follows for a monodimensional problem:

$$
\hat{\theta}_{[k]}= \begin{cases}Q_{[k]} / R_{[k]}, & \text { if } R_{[k]}>\varepsilon_{0} \\ \hat{\theta}_{[k-1]}, & \text { if } R_{[k]} \leq \varepsilon_{0}\end{cases}
$$

with

$$
\begin{aligned}
& R_{[k]}=\sum_{j=k-N_{L S}+1}^{k}\left(\beta^{(k-j)} \Phi_{[j]}^{T} \Phi_{[j]}\right) \\
& Q_{[k]}=\sum_{j=k-N_{L S}+1}^{k}\left(\beta^{(k-j)} \Phi_{[j]} Y_{[j]}\right) .
\end{aligned}
$$

A threshold value $\varepsilon_{0}$ is defined to fix a boundary on the smallest value of $R_{[k]}$ that can be taken into account. $\varepsilon_{0}$ guarantees that enough out-of-plane motion has been performed between two successive iterations of the algorithm to update the out-ofplane gradient information. $\varepsilon_{0}$ is such as $\varepsilon_{0}=\delta_{0}^{2}$, where $\delta_{0}$ is the estimated minimal distance from which the intensity variation of one pixel is relevant with respect to the image noise specific to the US sensor. Typically, we chose $\delta_{0}$ about the size of one pixel.

\section{B. Benefits of the 3-D Probes}

For the intensity-based visual servoing, the advantage of the 3 -D probes is to directly provide out-of-plane information that can be used in the visual features vector or to compute the interaction matrix. Depending on the technology of the 3-D probe, two different approaches are considered.

1) 3-D Approach: A matrix array 3-D probe provides a 3-D US volume in real time. In this case, the ROI considered in the visual servoing strategy is a set of voxels, whose intensities are used as visual information

$$
\mathbf{s}=\left(I_{1,1,1}, \ldots, I_{u, v, w}, \ldots, I_{M, N, L}\right)
$$

where $M, N$, and $L$ are, respectively, the width, height, and depth of the volume. With the modeling of the interaction matrix described in Section III-B, we can write the new interaction matrix associated with the intensity of one voxel $V(u, v, w)$ as

$$
\mathbf{L}_{I_{u, v, w}}=\nabla I_{(u, v, w)}\left[\mathbf{I}_{3} \quad-\left[{ }^{p} \mathbf{x}_{V}\right]_{\times}\right]
$$

with $\nabla I_{(u, v, w)}$ the 3-D image gradient of the voxel $V$ and ${ }^{p} \mathbf{x}_{V}=(x, y, z)$ the coordinates of $V$ in the probe frame. Given $\left(u_{0}, v_{0}, w_{0}\right)$ the voxel coordinates of the probe frame origin and $s_{z}$ the voxel depth size, we have

$$
\left(\begin{array}{l}
x \\
y \\
z
\end{array}\right)=\left(\begin{array}{c}
s_{x}\left(u-u_{0}\right) \\
s_{y}\left(v-v_{0}\right) \\
s_{z}\left(w-w_{0}\right)
\end{array}\right) .
$$

In particular, $z$ is no more equal to zero, and the $1 \times 6$ interaction matrix associated with the visual feature $I_{u, v, w}$ is then

$$
\begin{aligned}
& L_{I_{u, v, w}}=\left[\begin{array}{llll}
\nabla I_{x} & \nabla I_{y} & \nabla I_{z} & y \nabla I_{z}-z \nabla I_{y}
\end{array}\right. \\
& \left.-x \nabla I_{z}+z \nabla I_{x} \quad x \nabla I_{y}-y \nabla I_{x}\right] .
\end{aligned}
$$

Using the complete volume provided by the 3-D probe, the current interaction matrix can be computed online by filtering this volume with the 3-D derivative filters previously described (see Fig. 3).

2) 2-D Approach: The current limitation of the matrix array probes is their small field of view (FOV) and the low quality of the obtained volume. On the contrary, motorized 3-D probes offer a good quality of images, but a tradeoff is required between the size of the out-of-plane FOV and the probe frame rate. For instance, with a motor step of $1.4^{\circ}$, a volume of $28^{\circ} \mathrm{FOV}$ is reconstructed from a set of 20 images at a frame rate of $3 \mathrm{vol} / \mathrm{s}$. To increase this frame rate and perform real-time control of the probe, we propose to consider a 2-D approach. The visual information is extracted from one image plane and the 3-D information is only used for the image gradient computation. In this case, we do not need to capture a complete 3-D US volume but only two or four additional images that are acquired with the motorized sweeping of the 2-D transducer (see Fig. 4) at a frame rate of $12 \mathrm{vol} / \mathrm{s}$.

Since a motorized 3-D probe does not acquire parallel images due to the rotation angle $\alpha$ (see Fig. 4), new 3-D derivative filters are proposed in this section to take into account the elevation distance between the image pixels.

Given $C\left(u_{c}, v_{c}\right)$, the point corresponding to the US beams intersection. The 3-D filter applied to the pixel $P(u, v)$ of the current image $I_{0}$ corresponds to a set of three 2-D filters, respectively, applied to $P_{a}\left(u_{a}, v_{a}\right), P(u, v)$, and $P_{b}\left(u_{b}, v_{b}\right)$, where $P_{a}$ and $P_{b}$ are the image points of $I_{a}$ and $I_{b}$ that orthogonally project onto $P$ in $I_{0}$. Their coordinates are computed from the coordinates of $P$ and the angle $\alpha$ :

$$
\left\{\begin{array}{l}
u_{a}=u_{b}=u \\
v_{a}=v_{b}=\frac{\left(v+v_{c}\right)}{\cos (\alpha)}-v_{c} .
\end{array}\right.
$$

With the respective orientations of the acquired US images, the elevation distance between $P$ and $P_{a}$ or $P$ and $P_{b}$ is dependent on the pixel ordinate $v$ in the image. Weights inversely 


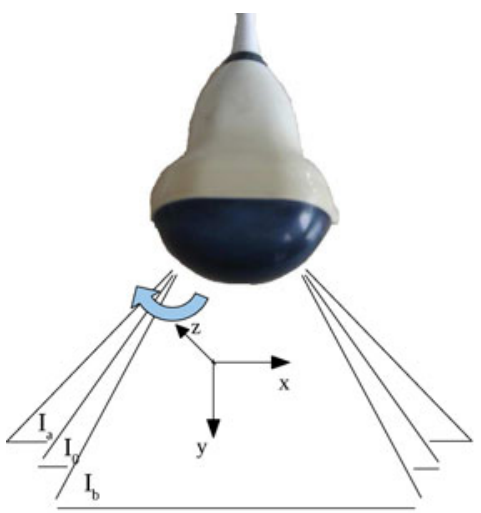

(a)

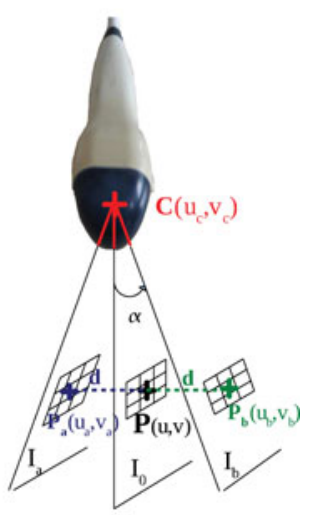

(b)
Fig. 4. (a) Additional US images provided by the 3-D probe are no longer parallel to the current one but are obtained after a rotation of an angle $\alpha$ around the image $x$-axis. (b) Derivative filters are weighted to take into account the variable distance $d$.

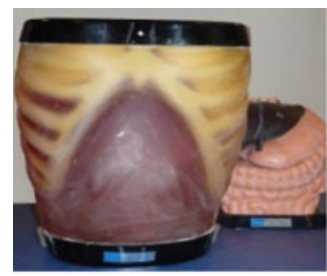

(a)

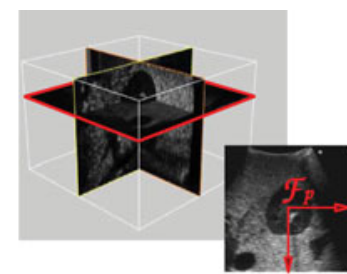

(b)
Fig. 5. (a) US abdominal phantom AB-41900-030 Kyoto Kagaku - ABDFAN. (b) Volume loaded in the simulator is represented by two orthogonal slices and the virtual probe plane, which is defined with the frame $\mathscr{F}_{p}$, is displayed in red.

proportional to this elevation distance are defined as follows:

$$
w(v)=\frac{1}{d(v)}=\frac{1}{\left(v+v_{c}\right) \tan (\alpha)} .
$$

These weights are multiplied to the coefficients applied to the images $I_{a}$ and $I_{b}$ with the nonweighted derivative filters (see Fig. 3).

\section{Simulation VALIDATION}

To validate the US intensity-based approach, we use a software simulator that we have developed to reconstruct and display a dense volume from a set of parallel images. Positioning and tracking tasks are then performed in this simulation environment to assess the advantages and the limits of our approach, respectively using 2-D and 3-D probes.

\section{A. Simulation Environment}

The simulator is built from the Visualization ToolKit (VTK) library [20] and the Visual Servoing Platform (ViSP) [21], both being open source $\mathrm{C}++$ libraries. The US simulator provides an external view of the loaded US volume by means of two central orthogonal slices as shown in Fig. 5. Moreover, in addition to this display functionality, the simulator allows the control of a virtual US probe and generates the internal view of this probe by a cubic interpolation process.

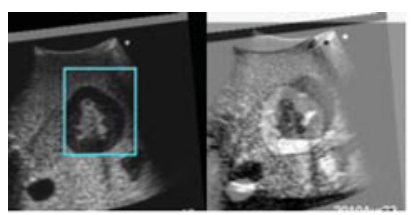

(a)

(b)

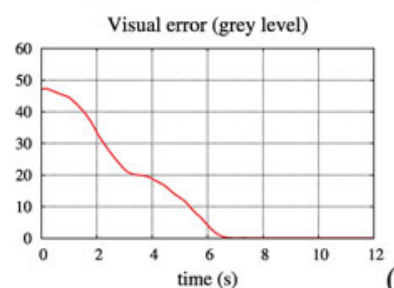

(e)

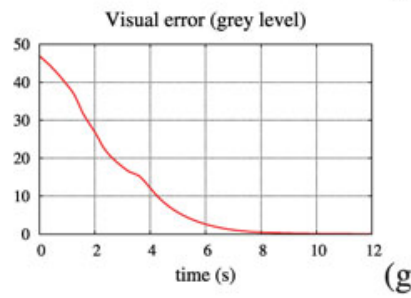

Visual error (grey level)

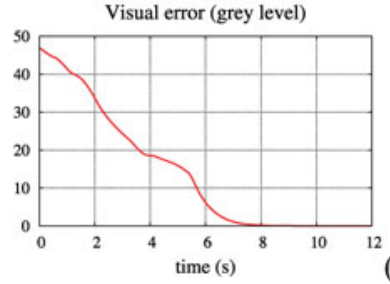

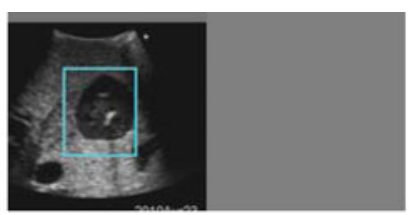

(c)

(d)
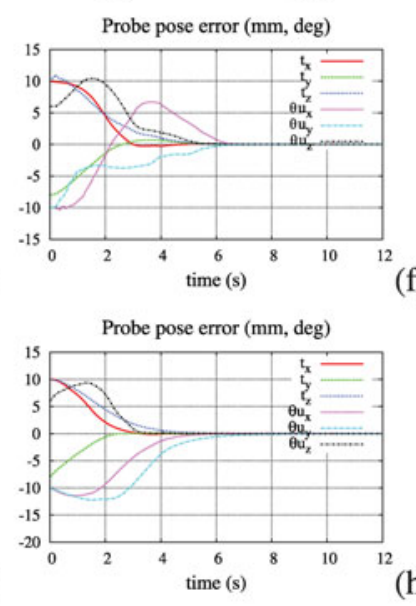

(h)

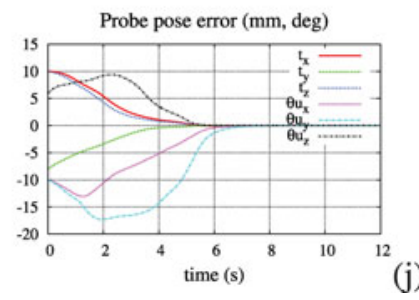

Fig. 6. (a) Visual convergence from (a) the initial US view to (c) the final one is shown by the corresponding difference image with the desired view [respectively, (b) and (d)]. Under the same simulation conditions, we compare the different solutions proposed to compute the image gradient with the nonrecursive estimation algorithm (e), (f) and with the weighted (g), (h) and nonweighted (i), (j) derivative filters. These latter curves correspond to the results obtained with a 3-D probe (see Section IV-D2).

For the simulation validation, we use the US volume of a kidney acquired on a realistic abdominal phantom (see Fig. 5). This volume is created from a set of 335 parallel images of size $250 \times 250$ and pixel size of $0.6 \mathrm{~mm} \times 0.6 \mathrm{~mm}$, which were automatically acquired using elevation intervals of $0.3 \mathrm{~mm}$.

\section{B. Positioning Tasks}

We first simulate a positioning task, using the simulation environment to obtain a ground truth of the evolution of the pose error of the US probe. We position the virtual probe in the simulator, and we consider the corresponding image as the desired one $I^{*}$. Then, the probe is moved away to a new pose where the observed organ section is considered as the initial image and the visual servoing is launched. In the pose expression, the $\theta \mathbf{u}$ representation is considered to describe the orientation, where $\mathbf{u}=\left(\begin{array}{lll}u_{x} & u_{y} & u_{z}\end{array}\right)^{\top}$ is a unit vector representing the rotation axis, and $\theta$ is the rotation angle.

The results of several positioning tasks are gathered in Fig. 6. The same control law (1) is applied to the probe with a gain $\lambda=1$, which is empirically adjusted to ensure the best behavior 
of the control law, and different methods for the computation of the 3-D gradient are compared.

The view of the virtual probe is shown at its (a) initial and (c) final positions. The cyan rectangle defines the ROI of size $100 \times 150$ pixels. To display the visual error between the current $I$ and desired $I^{*}$ images during the positioning task, a difference image $I_{\text {diff }}$ is computed as

$$
I_{\text {diff }}=\frac{\left(I-I^{*}\right)+255}{2} .
$$

The difference images corresponding to the initial view of the probe and to the final one are given in Fig. 6(b) and (d). The uniform gray color of this difference image after the convergence of the algorithm demonstrates the success of the positioning task since the final image perfectly coincides with the desired one. Moreover, we define a visual error function $\mathscr{C}$ to visualize the evolution of the error between the current and desired features vectors during the visual servoing

$$
\mathscr{C}=\sqrt{\frac{\left(\mathrm{s}-\mathrm{s}^{*}\right)^{\top}\left(\mathrm{s}-\mathrm{s}^{*}\right)}{N_{\mathrm{pix}}}} .
$$

$\mathscr{C}$ is expressed in gray levels and normalized by the number of pixels $N_{\text {pix }}$ in the considered ROI.

1) Online Gradient Estimation: In this positioning task, the in-plane gradient components $\nabla I_{x}$ and $\nabla I_{y}$ are computed in the current image with 2-D derivative filters and the out-of-plane component is estimated with the online algorithm. During the five first iterations of the algorithm, an open-loop translation is applied to the probe to initialize the estimation algorithms. Then, the value of the parameter $\nabla I_{z}$ is updated each time enough out-of-plane motion is applied to the probe. The results of the positioning task, presented in Fig. 6, show (e) the visual convergence and (f) the pose convergence of the control law. From an initial error $\Delta \mathbf{r}(\mathrm{mm} / \mathrm{deg})=(10,-8,10,-10,-10,6)$, the final pose error of the probe is less than $0.1 \mathrm{~mm}$ in translation and $0.1^{\circ}$ in rotation.

2) Derivative Gradient Filters: The curves (g) and (h) correspond to the use of a virtual motorized 3-D probe. In this case, the visual information is extracted from one image plane, and the 3-D geometry allows the acquisition of additional images to compute online the 3-D image gradient with derivative filters. The virtual 3-D probe provides at each iteration one current image along its plane and four additional images tilted with an angle $\pm \alpha= \pm 1.4^{\circ}$ and $\pm 2 \alpha= \pm 2.8^{\circ}$. The 3-D image gradient is directly computed from this set of images with three weighted $5 \times 5 \times 5$ filters, which avoids the initial out-of-plane motion of the probe required with the 2-D approach. The performance of the weighted derivative filters we have designed for a set of five non-parallel images is shown by comparison with the results obtained with the nonweighted filters [curves (i) and (j)]. Even if the desired pose is reached in both cases, with the same gain of the control law, the convergence is faster and more direct with the weighted filters.

3) Robustness to Calibration Errors and Images Noises: After the validation of the control law under ideal conditions, we introduce systemic and random errors likely to occur in the real robotic system. We take into account a calibration error between

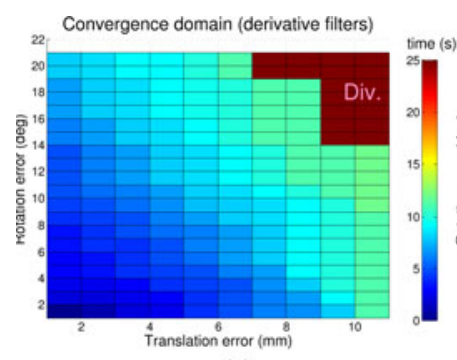

(a)

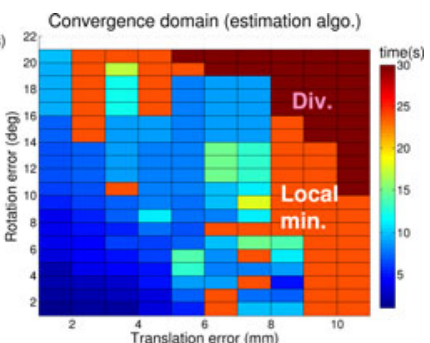

(b)
Fig. 7. Convergence domain of the intensity-based approach depends on the method of 3-D gradient computation. (a) With the derivative filters, the convergence time increases proportionally with the distance to the desired probe pose, and the divergence (in brown) is observed for an initial translation superior to $10 \mathrm{~mm}$ and an initial rotation superior to $14^{\circ}$. (b) With the estimation algorithm, local minimums (in orange) are often reached as soon as the initial pose is not very close to the desired one.

the robotic end-effector and the image frame of $5^{\circ}$ on each rotation and $5 \mathrm{~mm}$ on each translation. We also add normal Gaussian noises on the pixel intensities and on the pose measures with a variance of, respectively, three gray levels for the intensity and $3 \mathrm{~mm}$ and $1^{\circ}$ for the position and orientation measures. Under these simulation settings and considering the same desired pose of the probe, several positioning tasks are launched from different initial poses of the US probe in order to assess the convergence domain of the positioning task. The two methods of gradient computation (with derivative filters and with the estimation algorithm) are compared in Fig. 7, where the time of convergence is expressed as a function of the initial error of the probe.

As expected, with the gradient computation by image filters, the visual servoing approach is very robust to small calibration errors. Logically, the online estimation of the gradient is more sensitive to calibration errors and local minimums are regularly reached in this case when the initial pose error of the probe is superior to $5 \mathrm{~mm}$ for each translation and $8^{\circ}$ for each rotation.

4) 3-D Visual Information for a Larger Convergence Domain: In the previous simulations, only 2-D information, which is more generally available with US probes, was considered in the visual vector. However, the development of matrix-array 3D probes makes now possible the use of 3-D visual information in the control law according to the strategy presented in Section IV-B1. A new positioning task is presented in Fig. 8 where 20 parallel images are acquired in the simulation environment on both sides of the displayed view.

A volume of $80 \times 60 \times 41$ voxels is then considered in the control law and filtered with the 3 -D derivative filters (see Fig. 3) to compute the interaction matrix. To reach the same desired pose of the probe as done previously, a different initial pose is chosen, from which the one-plane algorithm falls in local minimum since it is farther than in the 2-D simulations

$$
\Delta r_{\text {init }}(\mathrm{mm}, \operatorname{deg})=(-10,12,-14,10,20,-14) .
$$

With the 3-D approach, the local minimum is avoided and the positioning task is well performed. We can, therefore, note that the 3-D information provided by the virtual 3-D probe ensures a larger domain of convergence of the visual servoing. 


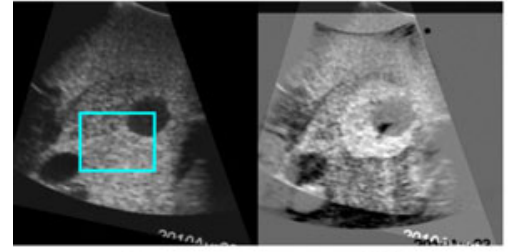

(a)

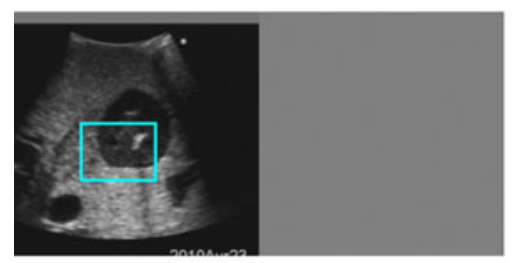

(d)

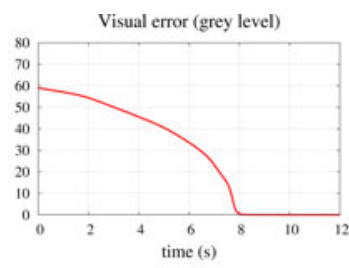

(c)

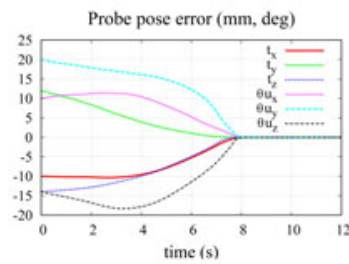

(f)
Fig. 8. Positioning task with a 3-D probe, which uses visual features extracted from a 3-D volume. The central view of the volume is displayed at the (a) initial and (d) final probe poses with the corresponding difference images (b), (e). (c) Visual and (f) pose convergence is displayed during the task.

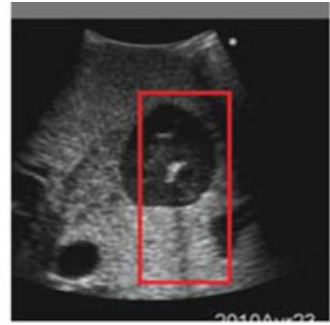

(a)

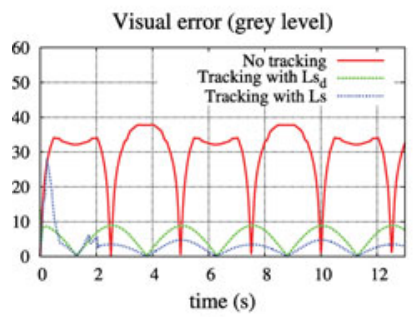

(c)

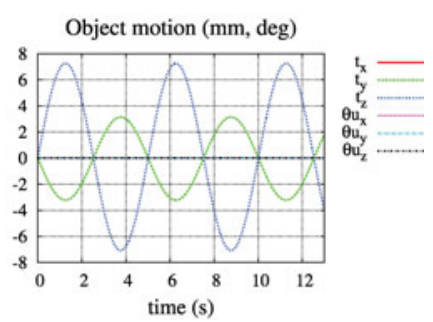

(b)

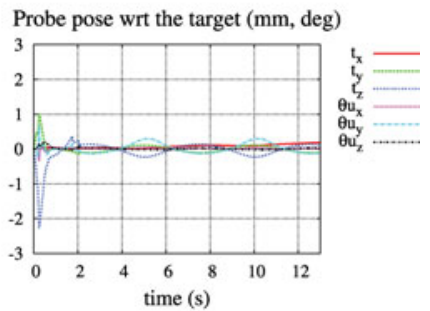

(d)
Fig. 9. Tracking task with a 2-D probe. (a) Stabilization of the desired US image (b) while the kidney volume undergoes a translational periodic motion. (c) Visual error is highly reduced with both methods, which corresponds to a low pose error (here displayed for the use of $\mathbf{L}_{\mathrm{s}}$ ) (d).

\section{Tracking Tasks}

The second robotic task we target is the active stabilization of a US image. In this case, the initial image is also the desired one, and the probe is moved to compensate for external motions applied to the observed object. In the following simulations, a sinusoidal motion of period $5 \mathrm{~s}$ and amplitude $A y=7 \mathrm{~mm}$ along the vertical translation and $A z=15 \mathrm{~mm}$ along the outof-plane translation is applied to the US volume of the kidney that has been loaded in the simulator. In parallel, the velocity $\mathbf{v}_{c}$ computed from the intensity-based control law (1) with $\lambda=1.5$ is applied to the US probe and the results obtained, respectively, with the current and desired interaction matrices are presented in Fig. 9.

\section{On-line estimation of $\nabla \mathrm{I}_{\mathrm{z}}$ (grey level / $\mathrm{mm}$ )}

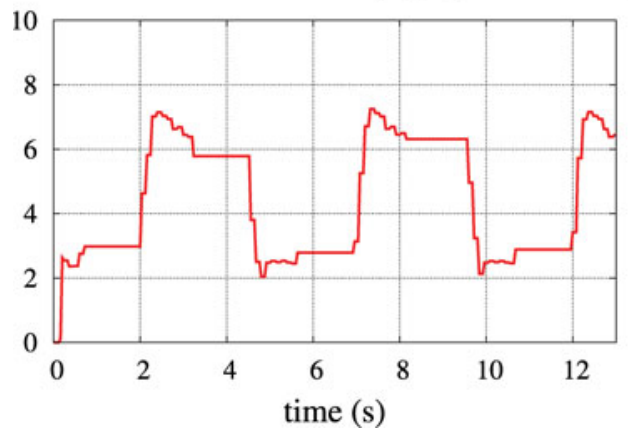

Fig. 10. Evolution of the out-of-plane value of the image gradient for one pixel of the ROI during the tracking task.

1) With Online Estimation of the Image Gradient: After the open-loop motion of the probe required to initialize the estimation algorithm, the current interaction matrix is computed with the image gradient components that are estimated by the nonrecursive estimation algorithm. In order to compute the variation of the parameter $\nabla I_{z}$ due to the probe motion and not to the disturbance motion, we set the least-squares window size to a small value $N_{\mathrm{LS}}=3$ and the forgetting factor to $\beta=0.9<1$. This temporal window size corresponds to a duration of $120 \mathrm{~ms}$ that is extremely short with respect to the $5 \mathrm{~s}$ of the disturbance period. By setting these parameters, the US volume can, therefore, be considered static during the $120 \mathrm{~ms}$ of the data acquisition. The stabilization is accurately performed since the maximum visual error is four gray levels (c) and the maximum pose error is $0.2 \mathrm{~mm}(\mathrm{~d})$.

We can note from Fig. 10 that the gradient is not updated continuously during the tracking. When the probe is perfectly synchronized with physiological motions (around $t=4 \mathrm{~s}$ for instance), the current and desired visual features are very close, and the velocity computed by the visual servoing control law, which depends to the visual error, is low. Therefore, the displacement is not sufficient to update the image gradient because of the threshold of (25). On the contrary, when some delay appears (around 5 and $10 \mathrm{~s}$ ), the probe velocity increases with the image error and the gradient image can be estimated.

2) With the Desired Interaction Matrix: The current pose of the probe being always close to its desired pose in this tracking task, the interaction matrix is well approximated by the desired interaction matrix [15]. This matrix, which is referred to as $\mathbf{L}_{\mathbf{s}^{*}}$, is computed once and for all at the initial pose without being updated during the servoing task. For this computation, five parallel images are acquired around the desired pose of the US probe and the 3-D derivative filters presented in Fig. 3 are applied to this set of images. The results obtained with $\mathbf{L}_{\mathbf{s}^{*}}$ are compared with the ones obtained with $\mathbf{L}_{\mathrm{s}}$ in terms of visual error (c). The pose error is very similar to the previous simulation, which validates the use of $\mathbf{L}_{\mathbf{S}^{*}}$ in this particular case.

\section{Conclusion}

Based on simulation validations, the 2-D intensity-based approach shows good results for local positioning tasks but is 


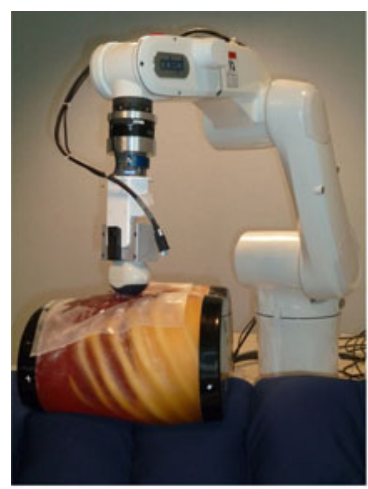

(a)

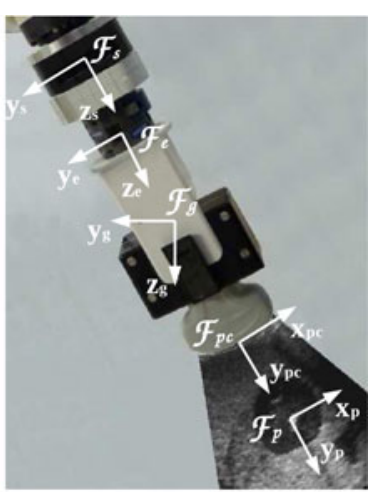

(b)
Fig. 11. (a) ADEPT Viper robotic system with a motorized 3-D US probe. (b) Robot end effector (frame $\mathscr{F}_{e}$ ) is equipped with a force sensor (frame $\mathscr{F}_{s}$ ) and a 2 -D US probe (frame $\mathscr{F}_{p}$ ).

generally sensitive to local minimums when considering further initial poses of the probe. In this case, the 3-D probes offer a better robustness to calibration errors in the computation of the interaction matrix and give more accurate results for positioning tasks from a remote initial pose, thanks to an increased convergence domain.

On the contrary, the 2-D probes are more fitted for tracking tasks than the 3-D ones thanks to their higher frame rate. If the tracking is accurate enough and the probe remains always close to the desired US view, the current interaction matrix is well approximated by the desired one $\mathbf{L}_{\mathbf{s}^{*}}$, which can be computed at the initial pose of the probe before the appearance of the disturbance. In the perspective of the breathing motion compensation, $\mathbf{L}_{\mathrm{s}^{*}}$ could be estimated when the patient holds his breath since this step requires no more than few seconds. However, if the dynamics of the system is not sufficient to track accurately the disturbance motion, then the online estimation of the current interaction matrix $\mathbf{L}_{\mathrm{s}}$ is necessary to compensate for the tracking delays (see Section VI-C).

\section{ROBOTIC EXPERIMENTS}

Experiments have been performed on a realistic US abdominal phantom, with an anthropomorphic robotic arm equipped with a US transducer and a force sensor [see Fig. 11(a)].

\section{A. Hybrid Vision/Force Control}

In the following experiments, we combine the visual control with a force control since the US probe is in contact with the surface of the phantom. Two sensors are then involved in an hybrid vision/force control based on an external control-loop approach [22]. The force control is dedicated to the control of the translational motion along the $y$-axis of the probe frame, while the five remaining dof are controlled by visual servoing.

1) Force Control: We implement a force control law to guarantee a constant resulting force of $1 N$ applied on the contact point $p c$ of the probe with the object surface along the $y$-axis of the probe frame. ${ }^{p c} H_{p c}$ corresponds to the contact force tensor expressed in the frame $\mathscr{F}_{p c}$, which is centered on the contact point and aligned with the probe frame $\mathscr{F}_{p}$ [see Fig. 11(b)]. This tensor is expressed as

$$
{ }^{p c} H_{p c}={ }^{p c} \mathbf{F}_{s}\left({ }^{s} H_{s}-{ }^{s} \mathbf{F}_{g}{ }^{g} H_{g}\right)
$$

where ${ }^{a} \mathbf{F}_{b}$ is a transformation matrix used to express, in the frame $\mathscr{F}_{a}$, a force tensor known in the frame $\mathscr{F}_{b}$ :

$$
{ }^{a} \mathbf{F}_{b}=\left[\begin{array}{cc}
{ }^{a} \mathbf{R}_{b} & \mathbf{0}_{3 \times 3} \\
{\left[{ }^{a} \mathbf{t}_{b}\right]_{\times}{ }^{a} \mathbf{R}_{b}} & { }^{a} \mathbf{R}_{b}
\end{array}\right]
$$

${ }^{a} \mathbf{t}_{b}$ and ${ }^{a} \mathbf{R}_{b}$ are the translation vector and the rotation matrix of the frame $\mathscr{F}_{b}$ with respect to the frame $\mathscr{F}_{a}$ and $\left[{ }^{a} \mathbf{t}_{b}\right]_{\times}$is the skew-symmetric matrix related to ${ }^{a} \mathbf{t}_{b}$.

${ }^{s} H_{s}$ is the total force tensor measured by the force sensor and ${ }^{s} \mathbf{F}_{g}{ }^{g} H_{g}$ is the gravity force applied to the force sensor due to the mass $m_{p}$ of the US probe, both are expressed in the force sensor frame. The ${ }^{g} H_{g}$ tensor is defined as ${ }^{g} H_{g}=$ $\left[\begin{array}{lllllll}0 & 0 & 9.81 m_{p} & 0 & 0 & 0\end{array}\right]^{T}$ in the frame $\mathscr{F}_{g}$ centered on the mass center of the probe as indicated in Fig. 11(b).

We express then the resulting force tensor in the probe frame $\mathscr{F}_{p}$, and we compute the instantaneous velocity of the probe $\mathbf{v}_{f}$ that is generated by the following proportional force control law:

$$
\mathbf{v}_{f}=-\frac{K^{p} \mathbf{F}_{p c}\left({ }^{p c} H_{p c}-{ }^{p c} H_{p c}{ }^{*}\right)}{k}
$$

where ${ }^{p c} H_{p c}{ }^{*}=\left[\begin{array}{llllll}0 & 1 N & 0 & 0 & 0 & 0\end{array}\right]^{T}$ is the desired contact force, $k$ is an estimate of the contact stiffness, and $K$ is the control gain.

2) Vision/Force Fusion: We use a $6 \times 6$ selection matrix $\mathbf{M}_{\mathrm{s}}=\operatorname{diag}(0,1,0,0,0,0)$ to apply the force control only along the $y$-axis of the probe. The complementary matrix $\left(\mathbf{I}_{6}-\mathbf{M}_{\mathbf{s}}\right)$ is then introduced to apply the vision control on the five remaining dof of the probe. To combine the force and the vision control, we send the following angular velocity $\dot{\mathbf{q}}$ to the end effector of the robotic arm:

$$
\dot{\mathbf{q}}={ }_{e} \mathbf{J}_{e}{ }^{-1} \mathbf{v}_{e}={ }_{e} \mathbf{J}_{e}{ }^{-1}{ }^{e} \mathbf{W}_{p}\left(\mathbf{M}_{\mathbf{s}} \mathbf{v}_{f}+\left(\mathbf{I}_{6}-\mathbf{M}_{\mathbf{s}}\right) \mathbf{v}_{p}\right)
$$

where ${ }_{e} \mathbf{J}_{e}{ }^{-1}$ is the inverse of the robot Jacobian and where ${ }^{e} \mathbf{W}_{p}$ is the transformation matrix that transforms a velocity skew from the probe frame to the effector frame:

$$
{ }^{e} \mathbf{W}_{p}=\left[\begin{array}{cc}
{ }^{e} \mathbf{R}_{p} & {\left[{ }^{e} \mathbf{t}_{p}\right]_{\times}{ }^{e} \mathbf{R}_{p}} \\
\mathbf{0}_{3 \times 3} & { }^{e} \mathbf{R}_{p}
\end{array}\right] .
$$

As we choose for safety reasons to give priority to the force control over the vision control, the latter can fail to converge to the desired image since the translational velocity component along the $y$-axis computed by the visual servoing control law is not applied to the probe. To deal with this issue, we apply this velocity to the ROI itself to readapt its position in the US image (see Fig. 12).

\section{B. Positioning Task With a 3-D Probe}

Up to now, the matrix-array 3-D probes have a small FOV and provide a volume of low resolution, particularly, difficult to process. Therefore, the positioning task is here performed with a 3-D motorized probe (4DC7-3/40, Ultrasonix) with a 


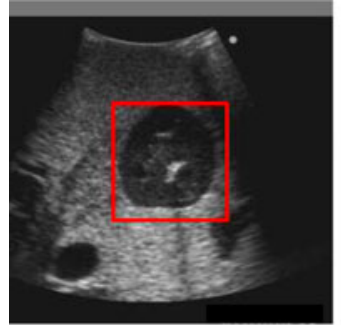

(a)

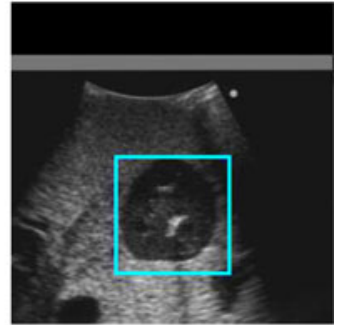

(b)
Fig. 12. Principle of the combination of the vision and force controls. (a) Target US slice with in red the desired ROI. (b) To oppose the vertical displacement of the probe due to the force control, the vision control is applied to the ROI, which is translated inside the US image.
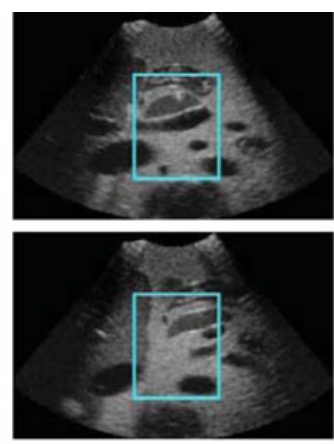

Visual error (grey level)

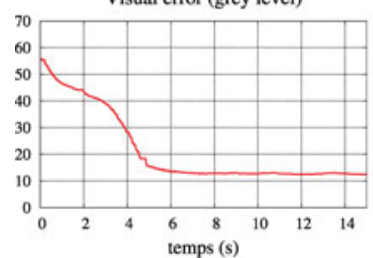

(a)
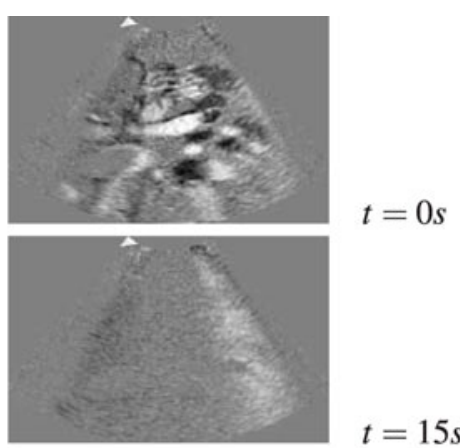

Pose error (mm, deg)

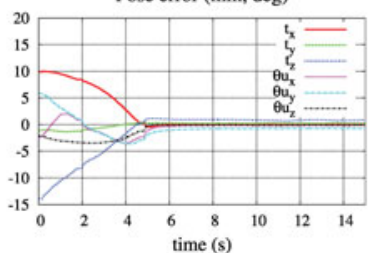

(b)
Fig. 13. Results of a positioning task with a 3-D probe. The central slice acquired by the probe is displayed at the beginning and the end of the task. (a) Visual error does not reach zero at convergence because of the noise of the sensor but the task is validated in terms of (b) pose error.

frequency bandwidth of 3 to $7 \mathrm{MHz}$ dedicated for abdominal imaging, using the 2-D approach detailed in Section IV-B2. With a depth of $12 \mathrm{~cm}$ and a motor step of $1.4^{\circ}$, this probe allows the acquisition of a small volume constituted of three images at a frame rate of $12 \mathrm{vol} / \mathrm{s}$. The control loop time is defined by this volume frame rate and at each iteration the 3-D image gradient is computed with the 3-D derivative filters. The gain of the control law is set to $\lambda=0.4$ and the results obtained with the abdominal phantom are given in Fig. 13.

The internal view of the probe and the difference image are shown at the initial $(t=0 s)$ and final $(t=15 \mathrm{~s})$ poses of the $3-\mathrm{D}$ probe. Only the central image acquired by the probe is displayed, the additional ones being only used to compute the 3-D image gradient. The visual convergence of the task can be seen on the uniform difference image corresponding to the final pose of the probe and on the curve (a) that corresponds to the evolution of the visual error during the positioning task. The pose convergence is also observed [see Fig. 13(b)], and the initial pose error
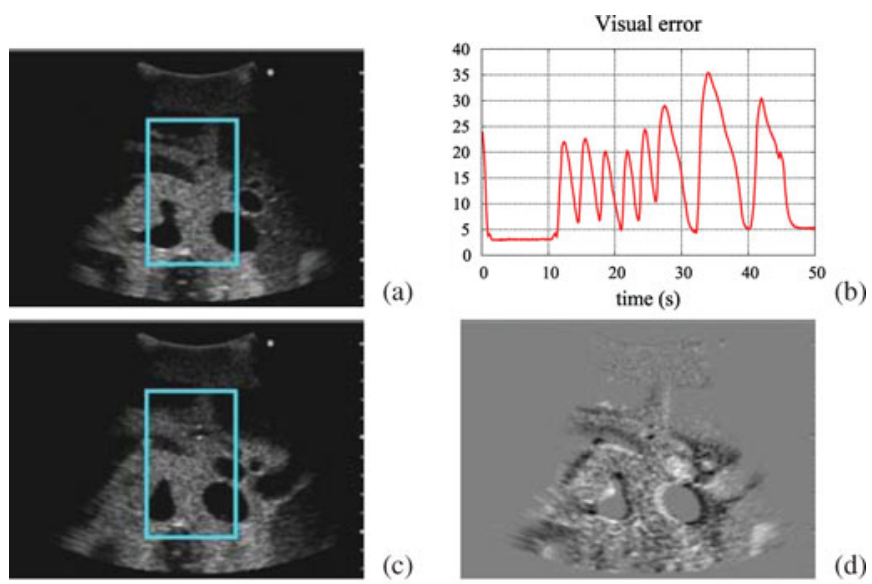

(b)

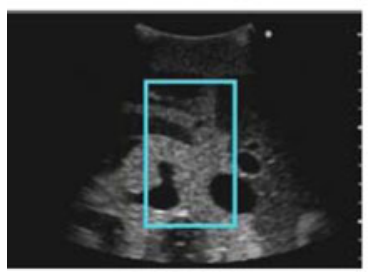

(c)

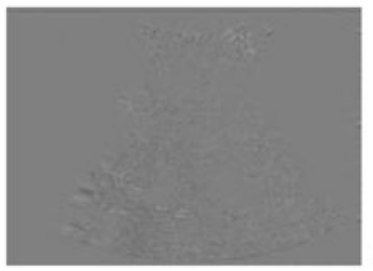

(d)

Fig. 14. Tracking of an abdominal cross section (a) with a 2-D probe. The tracking delay of the 2-D probe is observed through the evolution of the (b) visual error and through (c) the US image and (d) difference image corresponding to the maximum visual error (at $t=33 \mathrm{~s}$ ). Nevertheless, at the end of the disturbance, the probe reaches its desired pose as is shown by the (e) final US image and (f) difference image.

$\Delta r_{\text {init }}(\mathrm{mm}, \operatorname{deg})=(9.9,-1.1,-13.9,-2.4,5.8,-2.1)$ is minimized to $\Delta r_{\text {fin }}(\mathrm{mm}, \operatorname{deg})=(0.1,0.2,0.7,-0.1,-0.7,-0.1)$.

\section{Tracking Task of a Fast and Large Motion}

The robotic arm is now equipped with a 2-D convex US probe of 2-5 MHz frequency bandwidth (C60, Sonosite) to perform a tracking task. We position the 2-D US probe on the abdominal phantom, and we define the ROI in the US image [see Fig. 14(a)]. The force/vision control is launched after a small automatic back and forth out-of-plane translation used to initialize the estimation of the 3-D image gradient. Then, we manually apply various large and fast translational and rotational motions to the phantom. The dynamics of this disturbance exceeds the one of the control law in order to create important delays in the tracking and assess the ability of the control to overcome these delays. The tracking results are shown in Fig. 14.

In the current experiment where important probe pose errors appear due to the tracking delay, we update the interaction matrix during the tracking task thanks to the online estimation of the image gradient.

The interaction matrix, which is initialized at the desired pose, is then computed with the image gradient extracted directly from the current image (for the 2-D components $\nabla I_{x}$ and $\nabla I_{y}$ ) and estimated from the image measures as described in Section IV-A2 (for its out-of-plane component $\nabla I_{z}$ ). Despite the important disturbances applied to the phantom, the US probe follows the phantom motion and converges to the desired image when the motion stops. 


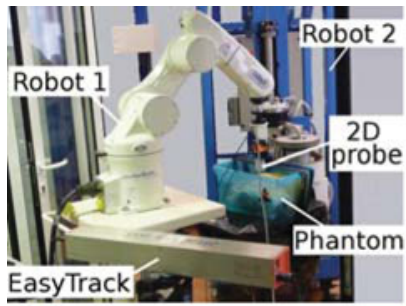

(a)

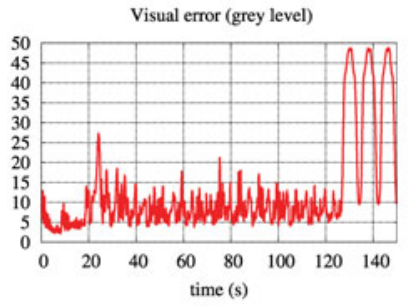

(c)

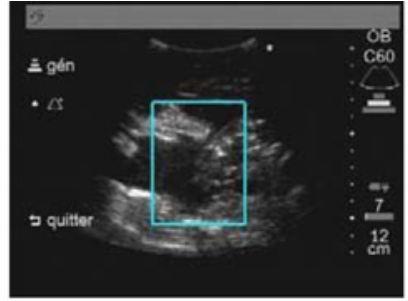

(b)

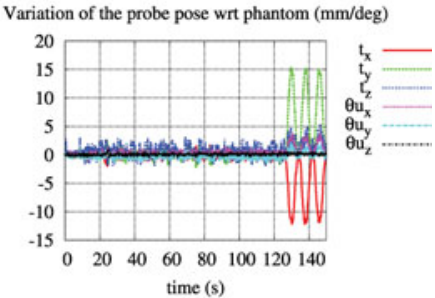

(d)
Fig. 15. Ex vivo results. (a) Experimental setup and (b) desired US image to stabilize. The tracking, launched at $t=0 \mathrm{~s}$ and stopped at $t=125 \mathrm{~s}$, ensures the compensation of the disturbance applied for $t>20 \mathrm{~s}$, as can be seen on the (c) visual error and (d) pose error.

\section{Tracking Task With a 2-D Probe: \\ First Ex Vivo Results}

For this validation, a chicken stuffed with pig liver and kidneys [see Fig. 15(a)] and immersed in a water tank to avoid air gaps inside its body is carried by a 6-dof robot (Robot2). A periodic motion is applied to this phantom with Robot2, while the tracking task is performed with a second robotic arm (Robot1) equipped with a 2D US probe. The probe frame rate is $25 \mathrm{im}$ ages/s and the control loop time is $40 \mathrm{~ms}$. Two optical markers are fixed on the probe and on the phantom and provide the relative pose of both elements thanks to an EasyTrack system. This relative pose is only used as a ground truth to validate the tracking task.

Robot1 is manually positioned above the phantom and the force control is applied with a force of $3 \mathrm{~N}$ to put the probe in contact with the chicken surface. A desired ROI is then defined in the US image, and a small back and forth motion is automatically realized to compute $\mathbf{L}_{\mathbf{s}^{*}}$ with a set of parallel images acquired around the desired image. A 3-D periodic motion along all translations and one rotation (around the probe axis) is then applied to the phantom. This disturbance has a period of $8 \mathrm{~s}$ and generates amplitudes of motion of $12 \mathrm{~mm}$ and $15 \mathrm{~mm}$ along horizontal axes and $10 \mathrm{~mm}$ along the vertical axis with a rotation of the phantom of $4^{\circ}$. To increase the tracking dynamics, we implement a Kalman filter to predict the target motion. The Kalman filter is based on a constant velocity model and takes as input the measures of the image features variation and the probe instantaneous velocity to provide an estimate of the target velocity. This estimated velocity $\hat{\mathbf{v}}_{\mathbf{o}}$ is finally reinjected into the control law (1) as a prediction term

$$
\mathbf{v}_{p}=-\lambda \mathbf{L}_{\mathbf{s}^{*}}^{+}\left(\mathbf{s}(t)-\mathbf{s}^{*}\right)+\hat{\mathbf{v}}_{\mathbf{o}} .
$$

The results of one tracking task with the 2-D probe are displayed in Fig. 15 for a gain $\lambda=0.4$. The disturbance motion is applied at $t=20 \mathrm{~s}$ and then at $t=125 \mathrm{~s}$, the compensation is stopped. The curve (c) shows the minimization of the visual error throughout the tracking task and the curve (d), which is obtained thanks to the EasyTrack system, validates the robotic task in terms of pose since the relative pose of the probe with respect to the phantom is maintained constant during the tracking $(t<125 \mathrm{~s})$.

In this experiment, the introduction of the Kalman filter in the control law improves the accuracy of the tracking task for sinusoidal motions with smooth changes of directions. For more complex motions, other predictive controllers should be considered, such as, for instance, a repetitive predictive controller $\mathrm{R}-\mathrm{GPC}[23]$ if the period of the disturbance is known.

\section{CONCLUSION AND PERSPECTIVES}

In this paper, a new approach has been proposed to control all the motions of a US probe by visual servoing. The originality of the approach is due to the direct use of the US B-mode image as visual information. In particular, no image processing or segmentation step is required to build the visual features vector that corresponds here to the intensity values of the pixels contained in a ROI of the current US image. The interaction matrix that is associated with these visual features is modeled and the proposed intensity-based approach is validated in simulation and with several robotic experiments where the US probe interacts with an abdominal phantom and animal soft tissue. 2-D and 3-D probes geometries are successively considered to perform positioning and tracking tasks. The 2-D approach shows good results for local positioning tasks and especially tracking tasks thanks to the high frame rate of the 2-D probe, while the 3-D probes offer a better robustness to calibration errors and give more accurate results for positioning tasks from a further initial pose.

So far, no in vivo validation of the intensity-based approach has been performed, and the current study is a first step that introduces and validates this new US visual servoing in experimental conditions, with slow and rigid motions to compensate. A further step will be to target a specific medical procedure to increase the robustness of the approach and deal with in vivo constraints. In this context, several medical applications have been identified that could benefit from the proposed approach.

With a tele-echography robot, the automatic positioning of the probe to a prerecorded US image and its tracking during the teleoperation can ease the diagnosis of a patient. The aim is to virtually stabilize an organ of interest with a robotic task based on the fusion of the vision and teleoperation controls. More generally, the active stabilization of an anatomic cross section could improve the diagnosis, based on US wave intensity measures, blood flow measures (Doppler), or enhanced imaging, that requires the observation of a stable anatomic target during several minutes and can be compromised by any motion of the patient. For the same reason, the measure of arteries dilation induced by blood flow, known as "Flow Mediated Dilation" [24] can benefit from the stabilization of the US image. Finally, surgical applications, such as the destruction of a kidney stone by high intensity focused US or the irradiation of tumors, are 
also targeted. The visual servoing approach can be used to follow the target and then maximize its irradiation while avoiding the healthy tissue.

\section{ACKNOWLEDGMENT}

The authors would like to thank P. Poignet and N. Zemiti for making available the robotic platform of LIRMM to conduct the experiments on animal soft tissue.

\section{REFERENCES}

[1] C. Nadeau and A. Krupa, "Intensity-based direct visual servoing of an ultrasound probe," in Proc. IEEE Int. Conf. Robot. Autom., Shanghai, China, May 2011, pp. 5677-5682.

[2] C. Nadeau and A. Krupa, "Improving ultrasound intensity-based visual servoing: Tracking and positioning tasks with 2-D and bi-plane probes," in Proc. IEEE/RSJ Int. Conf. Intell. Robot. Syst., San Francisco, CA, USA, Sep. 2011, pp. 2837-2842.

[3] J. Hong, T. Dohi, M. Hashizume, K. Konishi, and N. Hata, "A motion adaptable needle placement instrument based on tumor specific ultrasonic image segmentation," in Proc. 5th Int. Conf. Med.Imag. Comput. Comput.Assist. Interv., Tokyo, Japan, Sep. 2002, pp. 122-129.

[4] P. M. Novotny, J. A. Stoll, P. E. Dupont, and R. D. Howe, "Real-time visual servoing of a robot using three-dimensional ultrasound," in Proc. IEEE Int. Conf. Robot. Autom., Roma, Italy, Apr. 2007, pp. 2655-2660.

[5] M. A. Vitrani, H. Mitterhofer, N. Bonnet, and G. Morel, "Robust ultrasound-based visual servoing for beating heart intracardiac surgery," in Proc. IEEE Int. Conf. Robot. Autom., Roma, Italy, Apr. 2007, pp. 30213027.

[6] M. Sauvee, P. Poignet, and E. Dombre, "US image based visual servoing of a surgical instrument through non-linear model predictive control," Int. J. Robot. Res., vol. 27, no. 1, pp. 25-40, Jan. 2008.

[7] P. Abolmaesumi, S. Salcudean, W. Zhu, M. Sirouspour, and S. DiMaio, "Image-guided control of a robot for medical ultrasound," IEEE Trans. Robot., vol. 18, no. 1, pp. 11-23, Feb. 2002.

[8] D. Lee, N. Koizumi, K. Ota, S. Yoshizawa, A. Ito, Y. Kaneko, Y. Matsumoto, and M. Mitsuishi, "Ultrasound-based visual servoing system for lithotripsy," in Proc. IEEE/RSJ Int. Conf. Intell. Robot. Syst., Oct./Nov. 2007, pp. 877-882.

[9] R. Mebarki, A. Krupa, and F. Chaumette, "2-D ultrasound probe complete guidance by visual servoing using image moments," IEEE Trans. Robot., vol. 26, no. 2, pp. 296-306, Apr. 2010.

[10] N. Friedland and D. Adam, "Automatic ventricular cavity boundary detection from sequential ultrasound images using simulated annealing," IEEE Trans. Med. Imag., vol. 8, no. 4, pp. 344-353, Dec. 1989.

[11] C. Nadeau and A. Krupa, "A multi-plane approach for ultrasound visual servoing: Application to a registration task," in Proc. IEEE/RSJ Int. Conf. Intell. Robot. Syst., Taipei, Taiwan, Oct. 2010, pp. 5706-5711.

[12] C. Collewet and E. Marchand, "Photometric visual servoing," IEEE Trans. Robot., vol. 27, no. 4, pp. 828-834, Aug. 2011.

[13] A. Krupa, G. Fichtinger, and G. Hager, "Real time motion stabilization with B-mode ultrasound using image speckle information and visual servoing," Int. J. Robot. Res., vol. 28, no. 10, pp. 1334-1354, 2009.

[14] R. Nakadate, J. Solis, A. Takanishi, E. Minagawa, M. Sugawara, and K. Niki, "Out-of-plane visual servoing method for tracking the carotid artery with a robot-assisted ultrasound diagnostic system," in Proc. IEEE Int. Conf. Robot. Autom., Shanghai, China, May 2011, pp. 5267-5272.

[15] F. Chaumette and S. Hutchinson, "Visual servo control—Part I: Basic approaches," IEEE Robot. Autom. Mag., vol. 13, no. 4, pp. 82-90, Dec. 2006

[16] L. Mercier, T. Lango, F. Lindseth, and L. D. Collins, "A review of calibration techniques for freehand 3-D ultrasound systems," Ultrasound Med. Biol., vol. 31, no. 2, pp. 143-165, 2005.

[17] V. Acary and B. Brogliato, Numerical Methods for Nonsmooth Dynamical Systems: Applications in Mechanics and Electronics. New York, NY, USA: Springer-Verlag, p. 114, 2008.

[18] M. Hadwiger, J. M. Kniss, C. Rezk-salama, D. Weiskopf, and K. Engel, Real-time Volume Graphics. Boca Raton, FL, USA: CRC, 2006, pp. 112114.
[19] M. de Mathelin and R. Lozano, "Robust adaptive identification of slowly time-varying parameters with bounded disturbances," Automatica, vol. 35, no. 7, pp. 1291-1305, 1999.

[20] W. Schroeder, K. Martin, and B. Lorensen, The Visualization Toolkit: An Object Oriented Approach to 3-D Graphics, 3rd ed. Clifton Park, NY, USA: Kitware, 2003.

[21] E. Marchand, F. Spindler, and F. Chaumette, "ViSP for visual servoing: A generic software platform with a wide class of robot control skills," IEEE Robot. Autom. Mag., vol. 12, no. 4, pp. 40-52, Dec. 2005.

[22] J. De Schutter, T. De Laet, J. Rutgeerts, W. Decr, R. Smits, E. Aertbelin, K. Claes, and H. Bruyninckx, "Constraint-based task specification and estimation for sensor-based robot systems in the presence of geometric uncertainty," Int. J. Robot. Res., vol. 26, no. 5, pp. 433-455, 2007.

[23] J. Gangloff, R. Ginhoux, M. De Mathelin, L. Soler, and J. Marescaux, "Model predictive control for compensation of cyclic organ motions in teleoperated laparoscopic surgery," IEEE Trans. Contr. Syst. Technol., vol. 14, no. 2, pp. 235-246, Mar. 2006.

[24] M. C. Corretti, T. J. Anderson, E. J. Benjamin, D. Celermajer, F. Charbonneau, M. A. Creager, J. Deanfield, H. Drexler, M. GehardHerman, D. Herrington, P. Vallance, J. Vita, and R. Vogel, "Guidelines for the ultrasound assessment of endothelial-dependent flow-mediated vasodilation of the brachial artery," J. Amer. Coll. Cardiol., vol. 39, no. 2 pp. 257-265, 2002.

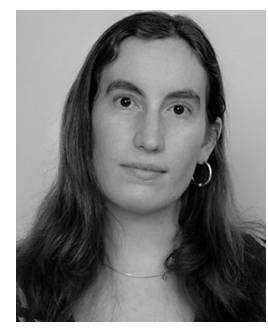

Caroline Nadeau received the Engineering degree in electrical engineering and automation from the $\mathrm{Na}-$ tional Polytechnic Institute of Toulouse, Toulouse, France, in 2008 and the Ph.D. degree in signal processing from the University of Rennes, Rennes, France, in 2011. Her Ph.D. research on medical robotics and visual servoing from ultrasound images was carried out with the Lagadic Group. INRIA Rennes-Bretagne Atlantique (IRISA), France.

Since 2012, she has been a Research Scientist with CEA List, Saclay, France, where she is currently a member of the Laboratoire Images, Tomographie et Traitement. Her current research interests include image-based robotics control, image processing, and computed tomography.

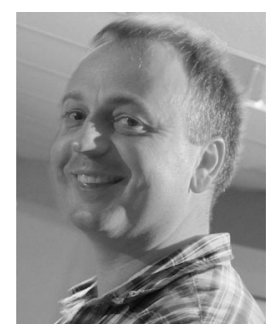

Alexandre Krupa (M'11) received the M.S. and $\mathrm{Ph} . \mathrm{D}$. degrees in control systems and signal processing from the National Polytechnic Institute of Lorraine, Nancy, France, in 1999 and 2003, respectively. He received the "Habilitation Á Diriger des Recherches" degree in signal processing from the University of Rennes, Rennes, France, in 2012. His $\mathrm{Ph} . \mathrm{D}$. research was carried out with the eAVR team (Control Vision and Robotics) with the Laboratoire des Sciences de l'Image de l'Informatique et de la Télédétection, Strasbourg, France.

From 2002 to 2004, he was an Assistant Associate Professor for undergraduate student lectures in electronics, control, and computer programming with Strasbourg University. Since 2004, he has been a Research Scientist with INRIA Rennes, France, where he is currently a member of the Lagadic Group. In 2006, he was a Postdoctoral Associate with the Computer Integrated Surgical Systems and Technology Engineering Research Center, Johns Hopkins University, Baltimore, MD, USA. His current research interests include medical robotics, computer-assisted systems in the medical and surgical fields, and, more specifically, the control of medical robots by visual servoing using ultrasound images. 